\title{
Pulsed KrF laser annealing of ZnS:Mn Laterally Emitting Thin Film Electroluminescent displays.
}

\author{
D.C. Koutsogeorgis*, E.A. Mastio, W.M. Cranton, C.B. Thomas \\ Department of Electrical and Electronic Engineering, The Nottingham Trent University, \\ Nottingham, Burton Street, NG1 4BU, UK.
}

\begin{abstract}
Pulsed KrF (248 nm) laser annealing was investigated as a post-deposition process for RF sputtered $\mathrm{ZnS}: \mathrm{Mn}$ phosphor layers used in laterally emitting thin film electroluminescent (LETFEL) displays. LETFEL devices consist of a phosphor layer sandwiched between two insulating thin films $\left(\mathrm{Y}_{2} \mathrm{O}_{3}\right)$, grown onto silicon substrates patterned with micro-mirrors $\left(\mathrm{SiO}_{2}\right)$. The micro mirror structure permits surface viewing by reflecting laterally emitted light due to internal waveguiding effects. Laser irradiation of the uncoated phosphor layer was performed using $\mathrm{KrF}$ excimer $248 \mathrm{~nm}$ laser pulses of $20 \mathrm{~ns}$ under an argon overpressure of 10.34 bars to limit laser ablation. The influence of the laser irradiation fluence on the LETFEL performance was investigated from 0.3 to $1.5 \mathrm{~J} / \mathrm{cm}^{2}$. Reported are the brightness-voltage characteristics of laser annealed, non-annealed and thermally annealed devices at $500^{\circ} \mathrm{C}$ for $\sim 1 \mathrm{~h}$. It is shown that the onset for light emission (threshold voltage) decreases with laser annealing. Using this novel method of annealing the brightness of LETFEL devices is observed to increase with increasing laser fluence.
\end{abstract}

Keywords: LETFELs, micro-mirrors, Laser annealing, electroluminescence, ZnS:Mn.

\section{Introduction}

Inorganic thin films of $\mathrm{ZnS}: \mathrm{Mn}$ have dominated the field of thin film electroluminescent devices (TFELs) $[1,2,3,4]$ being the core of alternating current thin film electroluminescent (ACTFEL) devices. Recently, the Displays Research Group at the Nottingham Trent University have developed a micromirror based structure for display devices, forming laterally emitting thin film electroluminescent (LETFEL) devices[5]. Critical to the fabrication of LETFELs, as for any TFEL device, is an annealing process that is carried out in order to activate the dopant in the phosphor thin

\footnotetext{
* Corresponding author: Tel: +44 115 8482176; Fax: +44 115 9486567; E-mail: demosthenes.koutsogeorgis@ntu.ac.uk The Nottingham Trent University, Dept of Electrical \& Electronic Engineering, Newton Bldg, Burton street, Nottingham NG1 4BU, UK.
} 
film, by effectively incorporating the luminescent dopant ions in the host lattice. This annealing process is conventionally carried out as a post-deposition thermal treatment in vacuum, typically at $500^{\circ} \mathrm{C}$ for $\sim 1$ hour. However previous work [6] has shown that thermal annealing of electroluminescent devices also results in a modification of the electron transport properties within the device, limiting the performance. In order to overcome the limitations that thermal annealing introduces, laser annealing has been proposed and following an initial period of investigation the optimum experimental configuration has been determined by successfully annealing ZnS:Mn thin films on $\mathrm{Si}$ [7]. In the present work we report the results of the first laser annealed LETFEL devices.

\section{Experimental details}

\subsection{LETFEL fabrication}

The LETFEL device, developed by the Displays Research Group at Nottingham Trent University, consists of a $\mathrm{ZnS}: \mathrm{Mn}$ phosphor layer sandwiched between two $\mathrm{Y}_{2} \mathrm{O}_{3}$ insulating thin films, grown onto silicon substrates patterned with micro-mirrors $\left(\mathrm{SiO}_{2}\right)$. Initially a thin film of $\mathrm{SiO}_{2}$ is deposited by PECVD onto 4 inch n-type Si wafers. Using photolithographic techniques the $\mathrm{SiO}_{2}$ layer is dry etched (plasma etched) to form the micro-mirrors, typically $1.5 \mu \mathrm{m}$ wide at the base and $1.5 \mu \mathrm{m}$ tall (Fig. 1). Then the 'sandwich' structure of two $300 \mathrm{~nm} \mathrm{Y}_{2} \mathrm{O}_{3}$ layers and a $800 \mathrm{~nm} \mathrm{ZnS:Mn} \mathrm{layer} \mathrm{are}$ deposited via RF magnetron sputtering. Prior to deposition of the second insulating layer the phosphor thin film is laser annealed. Finally, the top electrode is deposited and is dry etched to form the individual devices. This structure of electroluminescent devices permits surface viewing by reflecting laterally emitted light due to internal waveguiding effects and while allowing resolutions up to 1200 lines per inch.

Fig. 1. Schematic cross section of Laterally Emitting Thin Film Electroluminescent, LETFEL, device, showing the principle of reflected light emission.

In the past, aluminium has been used as the top electrode but for the present work Ti/W was also investigated. Aluminium, having a melting point $\left(660^{\circ} \mathrm{C}\right)$ close to the thermal annealing temperature, has been found to diffuse into the dielectric, leading to EL devices that break down easily. On the other hand, $\mathrm{Ti} / \mathrm{W}$ has a very high melting point (melting points are $\mathrm{Ti}: 1660^{\circ} \mathrm{C}$, $\mathrm{W}: 3410^{\circ} \mathrm{C}$ )[8], allowing thermal annealing after completing the fabrication (deposition, masking and plasma etch). Hence, with Ti/W as a top electrode both thermal and laser annealed LETFEL devices can be fabricated from the same wafer for direct comparison.

\subsection{KrF laser annealing}

For the purposes of laser annealing the experimental system illustrated in Fig. 2 was used.

Fig. 2. Optical bench for laser annealing with $\mathrm{KrF}$ laser.

The KrF laser (LAMBDA Physik) delivers $248 \mathrm{~nm}$ pulses of 20 ns duration. A variable number of Hoya plates (fused silica plates) are used to attenuate the beam. This results in the ability to provide different values of fluence. An excimer laser beam homogenizer (Exitech ltd., type EX-HS-700D) 
was used providing $80 \%$ deliverability and uniformity better than $2 \%$ throughout the laser spot. In this manner a spot size of $5 \times 5 \mathrm{~mm}$ is obtained with any fluence between 0.3 and $1.5 \mathrm{Joules} / \mathrm{cm}^{2}$. Two additional Hoya plates are introduced after the beam homogenizer to reflect a small portion of the beam to the on-line diagnostics: a photo-thermal converter (GENTEC ED-200) for monitoring the energy of each pulse and a CCD camera for monitoring the quality (uniformity) of the laser spot.

In order to have high performance laser annealing, ablation must be limited by housing the sample in a stainless steel pressure cell allowing high pressurized environments (150 psi) of an inert gas (Ar). For accurate positioning of the sample the cell is mounted on an X-Z translation stage, with motions driven by two stepper motors controlled by a computer.

\subsection{LETFEL device testing}

The LETFEL devices are tested in terms of electroluminescence, by measuring the luminance vs voltage characteristics. Measurements are performed on a probe station located in a dark enclosure and while driven by a sinusoidal wave the luminance is measured by coupling the emitted light to a photomultiplier tube via an optical fibre positioned $1 \mathrm{~cm}$ above the test device.

\section{Results}

In Fig. 3 the characteristic luminance vs voltage curves are shown, with the drive voltage at $5 \mathrm{Khz}$. For the laser annealed devices an increase of electroluminescence with increasing fluence of the laser annealing pulse is observed, With incident fluences $>1 \mathrm{~J} / \mathrm{cm}^{2}$, the laser annealed devices exhibit luminances in excess of that produced by the thermal annealed devices. Furthermore, the laser annealed devices can be driven at higher voltages than the thermal annealed without breaking down. Also the voltage threshold is found to decrease with increasing fluence of the annealing pulse.

Fig. 3. Characteristic luminance vs voltage curves of LETFEL devices with different annealing conditions.

\section{Conclusions}

For the first time electroluminescent devices based on micromirror structures have been successfully laser annealed. Using a single $20 \mathrm{~ns}$ pulse from a $\mathrm{KrF}$ laser, with a fluence above 1.0 $\mathrm{J} / \mathrm{cm}^{2}$, the luminance of the devices is greater than with conventional thermal annealing at $500^{\circ} \mathrm{C}$ for $\sim 1 \mathrm{~h}$ in vacuum. The lower threshold voltage for laser annealed devices could be attributed to some surface ablation. At the same time the laser annealed devices were found to be more stable than the thermally annealed. This technique is thus of interest both in terms of increasing luminance and device reliability.

\section{Acknowledgements}

The authors would like to thank EPSRC for financial support, and Dr G Hirst and his team at the Rutherford Appleton Laboratories for their expertise and assistance. 


\section{References}

[1] YA Ono, Encyclopedia Appl. Phys. 5, 295, 1993.

[2] K Okibayashi, T Ogura, K Terada, T Taniguchi, T Yamashita, M Yoshida and S Nakajima, Digest of 1991 SID International Symposium, 275.

[3] R Mach and GO Muller, Phys. Stat. Sol. (a), 69, 1982, 11.

[4] JA Ruffner, PF Hlava, RT Tuenge, SS Sun, PD Grandon, Thin Solid Films, vol. 310, no. 1, pp.123-131(9), 1997.

[5] CB Thomas, WM Cranton, R Stevens, SID International Symposium 96 Digest, 1996.

[6] WM Cranton, R Stevens, CB Thomas, AH Abdullah, MR Craven, Proc. IEE Colloquium on Materials for Displays, 1995.

[7] EA Mastio, WM Cranton, CB Thomas, E Fogarassy, S Unamuno, Appl.Surf.Sci. 139, 35, 1999.

[8] DR Lide (ed) in: CRC Handbook of Chemistry and Physics, 71st edition, 1990-1991, pp.4-35.

Figure legends.

Fig. 1. Schematic cross section of Laterally Emitting Thin Film Electroluminescent, LETFEL, device, showing the principle of reflected light emission.

Fig. 2. Optical bench for laser annealing with $\mathrm{KrF}$ laser.

Fig. 3. Characteristic luminance vs voltage curves of LETFEL devices with different annealing conditions. 


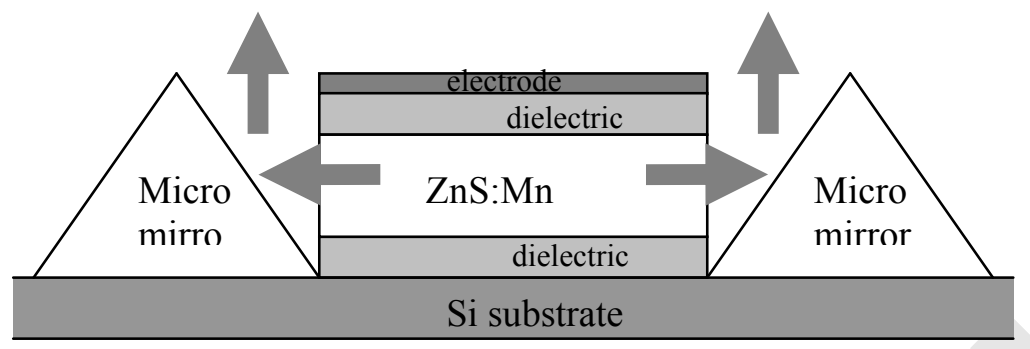

Fig. 1. D.C. Koutsogeorgis 


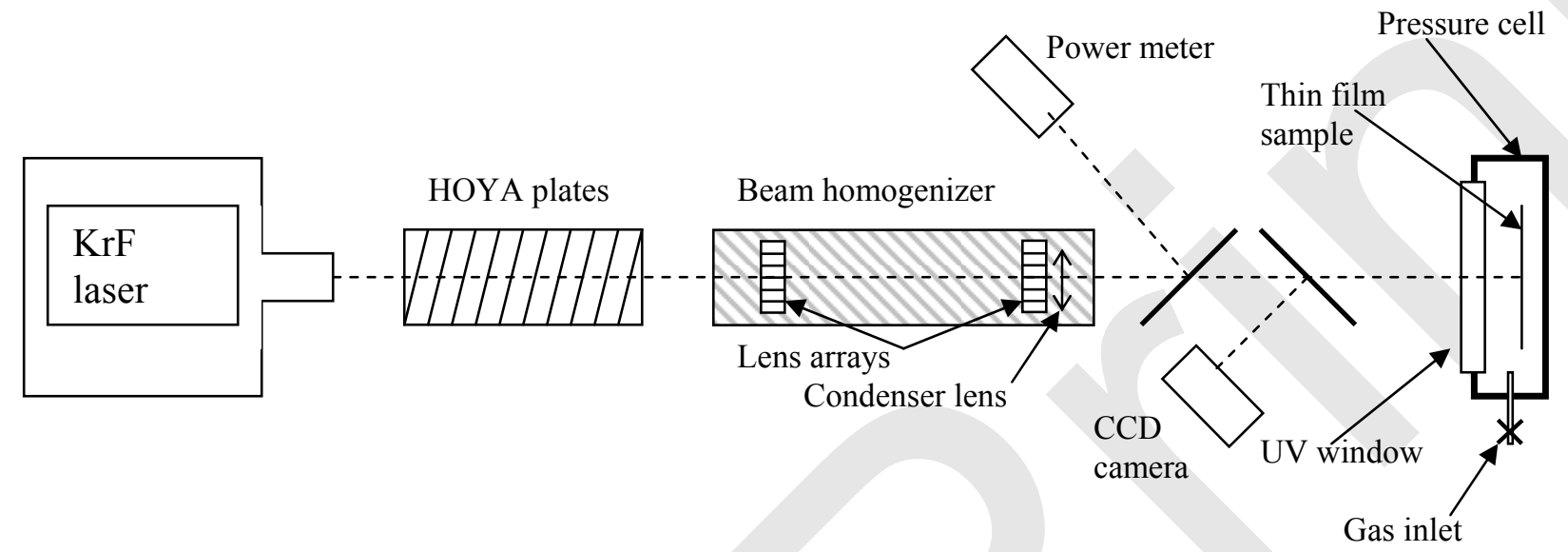

Fig. 2. D.C. Koutsogeorgis 


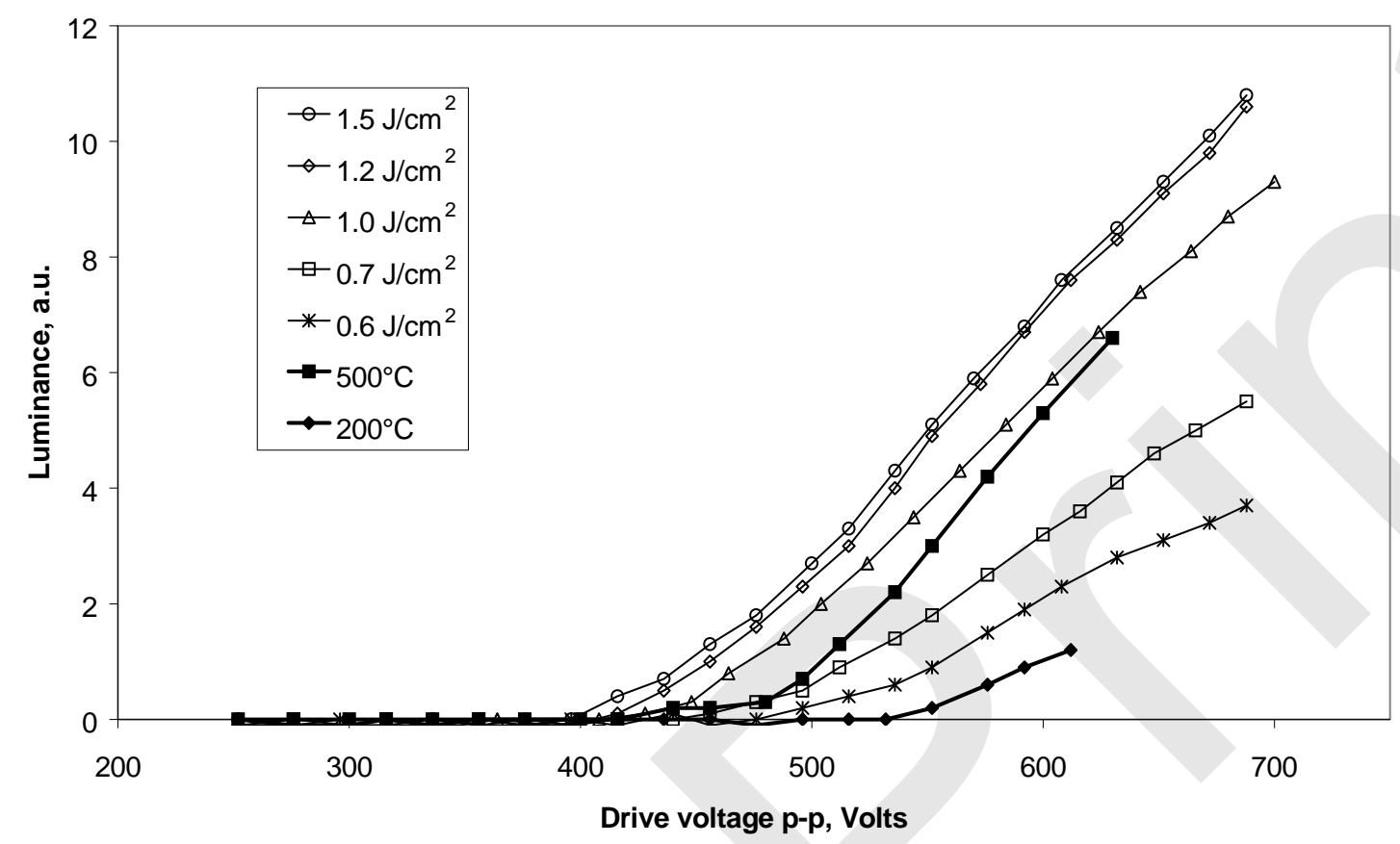

Fig. 3. D.C. Koutsogeorgis. 